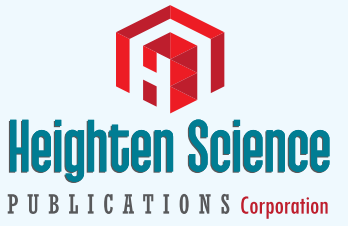

ISSN

2573-1726

\title{
Contractions of athlete's foot and its muscle oxygenation
}

\author{
A Paiziev* and F Kerimov \\ Uzbekistan State Institute of Physical Culture (USIPC), Uzbekistan
}

*Address for Correspondence: A Paiziev, Uzbekistan State Institute of Physical Culture (USIPC), Uzbekistan, Tel: +(998-71) 2470701; +(998-94) 6924422; Fax: +(998-71) 2628767; Email: adkhampaiziev@gmail.com

Submitted: 21 November 2018

Approved: 04 December 2018

Published: 05 December 2018

Copyright: (c) 2018 Paiziev A, et al. This is an open access article distributed under the Creative Commons Attribution License, which permits unrestricted use, distribution, and reproduction in any medium, provided the original work is properly cited

Keywords: Oxygenation; Skeletal muscle; Isometric exercise; Tissue oximeter

\section{Abstract}

Sustained isometric contractions of skeletal muscles produce intramuscular pressures that leads to blood flow restriction. In result an active muscle feels deficit of oxygen what bring to muscle fatigue. In another side during exercise we have physiological contradiction between raising of oxygen demand by working muscle and restriction of blood flow due to vessel pressing. To clarify this issue many research has been performed based mainly on measurement of blood flow in muscle tissue. The purpose of this study was to assess real-time changes in muscle oxygenation during a sustained isometric contractions of dorsiflexor muscle of low (30\%), moderate $(60 \%)$ and submaximal (90\%) intensity. Experiments were conducted using the subject's dominant (right) leg. Volunteers was recruited from eight male students of USIPC (age: $19 \pm 2$ years, weight: $75 \pm 6$ $\mathrm{kg})$. Tissue oxygenation index $\left(\mathrm{StO}_{2}\right)$ were recorded from the tibialis anterior using NIRS device (NONIN). Saturation was higher at $30 \%$ compared with both $60 \%$ and $90 \% \mathrm{MVC}$ at all time points after start exercise and higher at $60 \%$ than $90 \%$. Oxygen consumption $\left(\mathrm{VO}_{2}\right)$ permanently increased from slow $(30 \%)$ to moderate $(60 \%)$ and submaximal contractions. After cessation of the each contraction there was a large and immediate hyperemic response. Rate of $\mathrm{StO}_{2}$ increasing after effort cessation what reflects the resaturation of hemoglobin which depend on integrity and functionality of vascular system and reflects blood vessel vasodilation. $\mathrm{StO}_{2}$ restoration rate permanently increased from slow $(30 \%)$ to moderate $(60 \%)$ and submaximal contractions too. At last on final stage of experiment arterial occlusion test has been performed to determine the minimal oxygen saturation value in the dorsiflexors. Oxygen saturation reached a $24 \pm 1.77 \%$ what is significantly higher than $\mathrm{StO}_{2}$ after 60 and $90 \% \mathrm{MVC}$.

So, we can conclude that oxygen saturation at $60 \%$ and $90 \%$ MVC are similar and sharply decreased after start of exercise. It means that after $60 \% \mathrm{MVC}$ take place occlusion of blood vessels due to intramuscular pressure. Oxygen consumption of active muscle increased depend on intensity of exertion according to increasing of oxygen demand. $\mathrm{StO}_{2}$ resaturation rate $(\mathrm{Re})$ permanently increased from slow $(30 \%)$ to moderate $(60 \%)$ and to submaximal contractions. Re increasing after effort cessation reflects the resaturation of hemoglobin which depend on integrity and functionality of vascular system and reflects blood vessel vasodilation.

\section{Introduction}

Sustained isometric contractions (SIC) of skeletal muscles produce intramuscular pressures (IMP) that leads to muscle blood flow (MBF) restriction and limit its delivery to tissue [1]. MBF play key role to regulate intensity and type of muscle contractions [2], but SIC have considerable impact to MBF and in result a active muscle feels deficit of oxygen and nutrients what bring to muscle fatigue. In another side during exercise we have physiological contradiction between raising of oxygen demand by working muscle and blood vessel pressing due to IMP during muscle sustained isometric contraction. To clarify this issue many research has been performed investigations based mainly on measurement of blood flow in muscle tissue [3-6]. But in dynamic regime during exercise there are limited measurements of MBF by using Doppler ultrasound. Need to note, that direct measurements of hemodynamic response of working muscle possible by using fMRI and PET but it is not accessible especially in field training setting [7].

In another side difference methods indicate that complete occlusion of MBF during 
sustained muscle contractions take place at $50-60 \%$ of maximal voluntary contractions (MVC) $[3,4,6]$, but in [3] shown that blood flow was not occluded at the level of the conduit artery during any of the contraction intensities. In some of published papers mentioned about insensitivity of blood flow to the muscle contraction intensity. So, at present our understanding about hemodynamic response of skeletal muscle to wide range of muscle contraction (from low, moderate up to submaximal MVC) still remains limited.

Despite to advantages of above mentioned methods (fMRI, PET and Doppler ultrasound) only one paper in part has been devoted to measure hemodynamic response of contractile muscles to SIC [3] by using NIRS (near infrared spectroscopy) technology. Near-infrared spectroscopy (NIRS) is a well-established optical technique that monitors changes in concentration of the chromophores oxyhemoglobin $\left(\mathrm{O}_{2} \mathrm{Hb}\right)$, deoxyhemoglobin $(\mathrm{HHb})$, total hemoglobin and tissue oxygenation index $\left(\mathrm{StO}_{2}\right)$ in a variety of tissues $[8,9]$. NIRS utilizes the relative transparency of tissue to photons in the near-infrared (NIR) spectrum (700-900nm), and the oxygen-dependent absorption changes of these photons by hemoglobin $(\mathrm{Hb})$ and myoglobin $(\mathrm{Mb})$. NIRS instruments let us by using Lambert-Beer law and software algorithms to derive chromophore concentrations from raw optical data, and distinguish between oxy- and deoxy-hemoglobin/myoglobin. The majority of commercially available NIRS instruments are continuous wave (CW) spectrophotometers, and have proven reliability in the measurement of changes in $\mathrm{O}_{2} \mathrm{Hb}$ and $\mathrm{HHb}$. CW NIRS instruments have small size, particularly those with telemetric capacity, represent an important advance in sport science and exercise physiology studies. The non-invasive nature of the transcutaneous NIRS interface, and the ability to monitor continuously even during physical movement and active exercise provide an important means of measuring oxygenation and hemodynamics in muscle tissue during exercise. The purpose of this study was to assess real-time changes in muscle oxygenation during a sustained isometric contractions of dorsiflexor muscle of low, moderate and submaximal intensity.

\section{Materials and Methods}

\section{Subjects}

The dorsiflexor muscle was selected for this experiment. Experiments were conducted using the subject's dominant (right) leg. Volunteers was recruited from eight male not trained students of USIPC (age: $19 \pm 2$ years, weight: $75 \pm 6 \mathrm{~kg}$ ).Information about purpose and potential risks about the experiment was given to the subjects. All participants were given adequate instructions on the study, and the study was conducted with the approval of the institutional review board at the Uzbek State Institute of Physical Culture in Tashkent, Uzbekistan. Subjects were seated in a hand-made site with isometric torso-dynamometer (DC-200, Russia). Tissue oxygenation index $\left(\mathrm{StO}_{2}\right)$ were recorded from the tibialis anterior using NIRS device (Nonin's SenSmart ${ }^{\mathrm{TM}}$ Model X-100, USA). Sensor head were placed over the belly of the tibialis anterior muscle. All subjects was right handed.

\section{Protocol of experiment}

Over one day male volunteers performed dorsiflexor contractions by using handmade installation, torso-dynamometer and NIRS devise. The subject was seated for 15-20 min prior to the test. The right leg rested on a torso dynamometer with their ankle positioned at $30^{\circ}$ of plantar flexion. The maximum voluntary contraction (MVC) force of the subject was determined before the test and calculated 30\%, 60\% and 90\% MVC. Protocol of experiment consist on pre-exercise stage (rest) and three successive stages of sustained isometric contractions (30\%,60\% and 90\% MVC) lasted about 1 min and divided by rest period lasted 3 min (Figure 1). To determine the minimal oxygen saturation value in the dorsiflexors, were measured oxygenation parameters of dorsiflexor muscle in rest by using arterial occlusion test (at end of protocol of experiment). For this purpose a blood pressure cuff was placed around their right thigh to occlude blood flow to the 


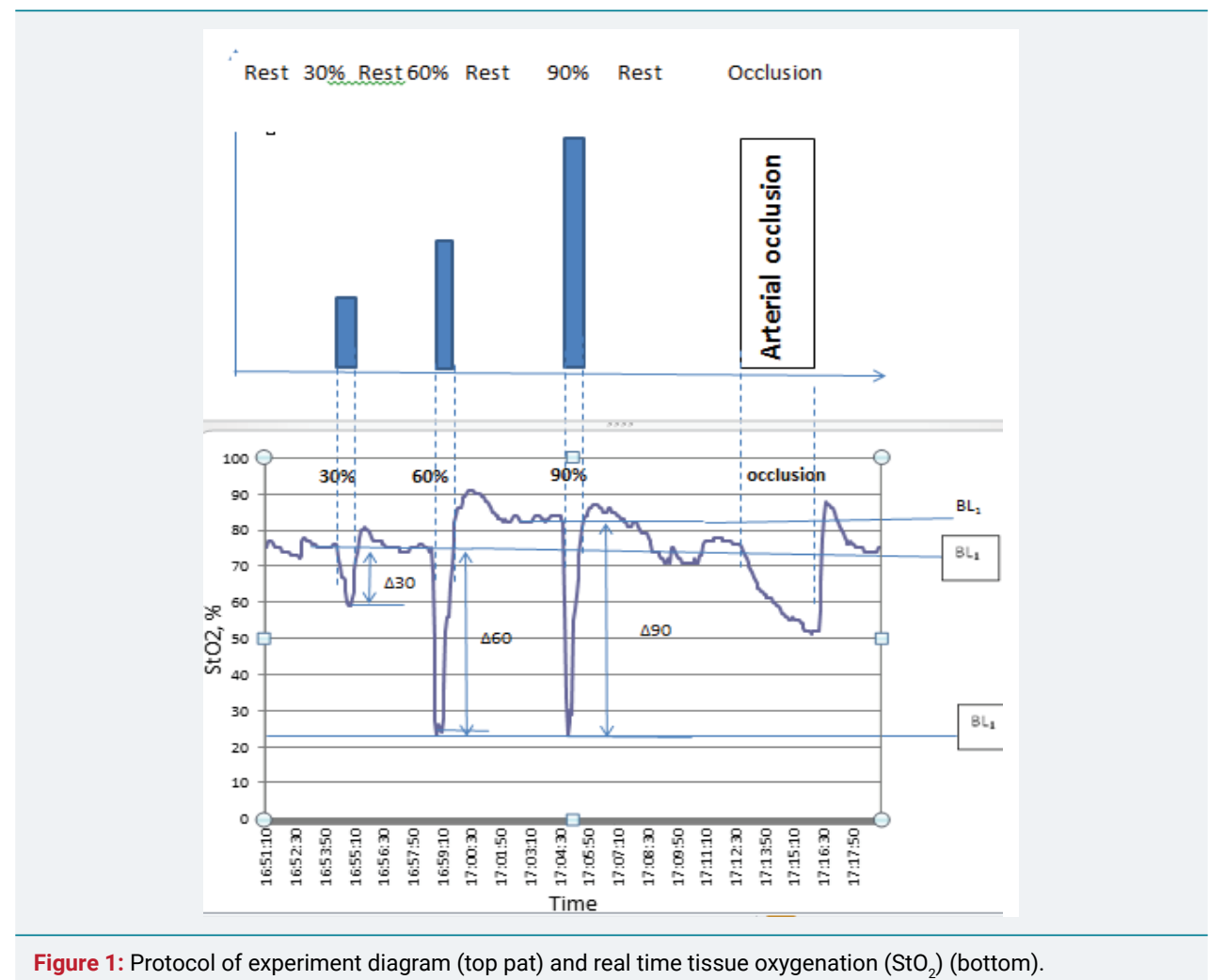

leg. Blood flow was occluded to the leg by inflating the cuff beyond $240 \mathrm{mmHg}$, and this occlusion was maintained until oxygen saturation researched a stable minimum level (last step of experiment, Figure 1). This way, both venous outflow and arterial inflow are blocked and systemic circulatory changes are sufficiently eliminated in the limb. Lacking the supply of well oxygenated blood, muscle metabolism fully depends on the available $\mathrm{O}_{2}$ in local capillaries and muscle cells. Depletion of local available $\mathrm{O}_{2}$ stores during arterial occlusion is monitored by NIRS as a decrease in $\mathrm{O}_{2} \mathrm{Hb}$ and a concurrent increase in $\mathrm{HHb}$ while tHb remains constant. A hyperaemic response can be observed after release of the arterial occlusion. Blood volume increases rapidly, resulting in a fresh pool of $\mathrm{O}_{2} \mathrm{Hb}$ and a quick wash-out of $\mathrm{HHb}$. Using the arterial occlusion method including the recovery phase, it is possible to calculate $\mathrm{O}_{2}$ consumption, reoxygenation rate and the half-recovery times of the signals [10].

\section{Statistics}

Differences in the $\mathrm{StO}_{2}$, desaturation rate (De) and resaturation rate (Re) between muscle contraction intensities were analyzed by the Student's t-test in the statistical software package "Statistica" for Windows (version 13). Differences of $\mathrm{P}<0.05$ were considered as statistically significant. All data are presented as mean \pm SD.

\section{Results and Discussion}

Maximal force was $18 \mathrm{~N}$ at the onset of the $100 \%$ MVC contraction. Calculated contraction intensities (F) for 30, 60 and 90\% MVC shown in table 1. Efforts of 30, $60 \%$ and $90 \%$ MVC were held constant throughout exercise.

The recovery baseline (BL) is the stable $\mathrm{StO}_{2}$ value that occurs during the rest period following test time. The recovery baseline is determined after a muscle contractions which will usually result in an increased $\mathrm{StO}_{2}$ value owing to increased physiological function. Here we can distinguish difference level of baseline recovery before and after each step of efforts. BL1 do not change after warm-up step (30\%MVC) (about 73\% $\mathrm{StO}_{2}$ ) but after moderate and submaximal contractions we have raised value BL2 plateau (78\%). The Performance Baseline is the minimum $\mathrm{StO}_{2}$ value reached during a exercise. 
Performance baseline of oxygen saturation during 30\% MVC have value about 30\% but after moderate and submaximal contraction achieved minimal level (20\%) (Table 1).

To comparison oxygen consumption under difference sustained isometric contractions on figure 2 shown dorsiflexor muscle oxygen saturation at different levels of maximal efforts.

Saturation was higher at 30\% compared with both 60 and 90\% MVC at all time points after start exercise and higher at 60 than $90 \%$ (Figure 2). Oxygen consumption (De, Table 1) permanently increased from slow (30\%) to moderate $(60 \%)$ and submaximal contractions. Separately De trends shown on figure 3.

After cessation of the each contraction there was a large and immediate hyperemic response. This response shown on figure 3 for three levels of MVC. Rate of $\mathrm{StO}_{2}$ increase after effort cessation reflects the resaturation rate of hemoglobin which depend on integrity and functionality of vascular system and reflects blood vessel vasodilation. $\mathrm{StO}_{2}$ resaturation rate permanently increased from slow (30\%) to moderate $(60 \%)$ and submaximal contractions (Table 1 and Figure 4).

At last on final stage of experiment (Figure 1) arterial occlusion test has been performed to determine the minimal oxygen saturation value in the dorsiflexors. Oxygen saturation reached a $24 \pm 1.77 \%$ what is significantly higher than $\mathrm{StO}_{2}$ after 60 and 90\%MVC (Table 1).

Table 1: Muscle tissue oxygenation parameters during difference level of sustaining isometric contractions and arterial occlusion test (AO). BL- baseline level of $\mathrm{StO}_{2}$, De-desaturation rate during exersice and $\mathrm{AO}$, Re-resaturation rate of $\mathrm{StO}_{2}$ after cesation efforts and $\mathrm{AO}, \Delta \mathrm{StO}_{2}$-difference between recovery and performance baseline level of $\mathrm{StO}_{2}$.

\begin{tabular}{|c|c|c|c|c|}
\hline MVC & $30 \%$ & $60 \%$ & $90 \%$ & AO \\
\hline $\mathrm{F},(\mathrm{N})$ & 5.4 & 10.8 & 16.2 & $77.75 \pm 3.46$ \\
\hline $\mathrm{BL},(\%)$ & $73.16 \pm 0.29$ & $73 \pm 0.87$ & $78.66 \pm 3.17$ & $-0.23 \pm 0.03$ \\
\hline $\mathrm{Re},(\% / \mathrm{sec})$ & $-1.06 \pm 0.09$ & $-4.19 \pm 0.16$ & $-4.80 \pm 0.16$ & $2.5 \pm 0.08$ \\
\hline$\Delta \mathrm{StO}_{2}(\%)$ & $0.84 \pm 0.19$ & $1.54 \pm 0.25$ & $2.65 \pm 1.44$ & $24 \pm 1.77$ \\
\hline
\end{tabular}

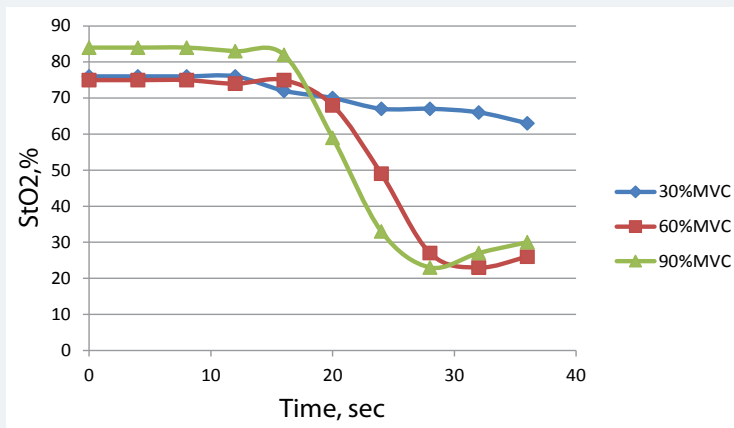

Figure 2: Dorsiflexor muscle oxygen saturation during sustained isometric contractions under $30 \%, 60 \%$ and $90 \%$ of MVC

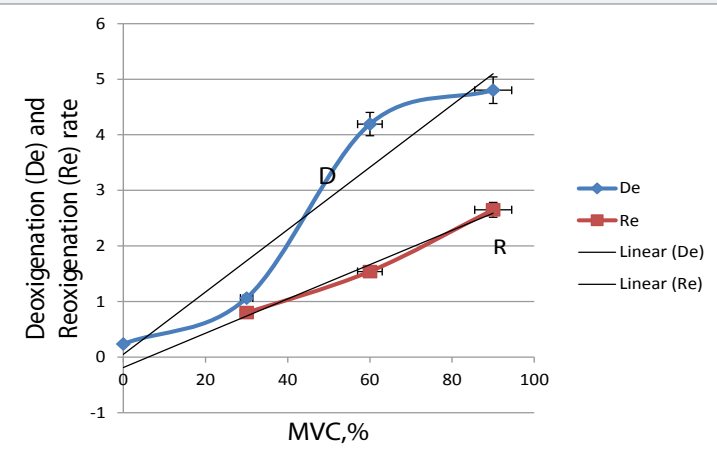

Figure 3: Deoxygenation and reoxygenation rate depend on MVC 


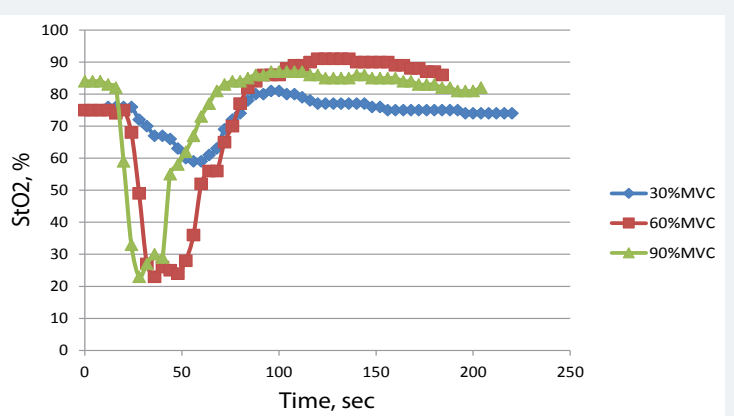

Figure 4: Dorsiflexor muscle oxygen saturation during difference level of SIC , recovery and reactive hyperemia phases.

\section{Conclusion}

- The recovery baseline (BL) after exercise increased due to increased physiological physiological state of muscle.

- The Performance Baseline is the minimum $\mathrm{StO}_{2}$ value reached during a exercise. Performance baseline of oxygen saturation increased after moderate and submaximal efforts and achieved its plateau.

- Oxygen saturation at 60 and 90MVC are similar and sharply decreased after start of exercise. It means that after $60 \%$ MVC take place occlusion of blood vessels due to intramuscular pressure.

- Oxygen consumption of active muscle increased depends on intensity of exercise according to increasing of oxygen demand.

- $\mathrm{StO}_{2}$ resaturation rate (Re) permanently increased from slow (30\%) to moderate $(60 \%)$ and to submaximal contractions. Re increasing after effort cessation reflects the resaturation of hemoglobin which depend on integrity and functionality of vascular system and reflects blood vessel vasodilation.

\section{Acknowledgement}

The authors express their gratitude to the Swiss National Science Foundation to support this work via grant no. IZ74Z0_137423 (S-84301-05-01).

\section{References}

1. Degens $\mathrm{H}$, Salmons $\mathrm{S}$, Jarvis JC. Intramuscular pressure, force and blood flow in rabbit tibialis anterior muscles during single and repetitive contractions. Eur J Appl Physiol Occup Physiol. 1998; 78: 13-19. Ref.: https://goo.gl/eUqRUj

2. Saltin B, Radegran G, Koskolou MD, Roach RC. Skeletal muscle blood flow in humans and its regulation during exercise. Acta Physiol Scand. 1998; 162: 421-436. Ref.: https://goo.gl/ge49wk

3. McNeil CJ, Allen MD, Olympico E, Shoemaker JK, Rice CL. Blood flow and muscle oxygenation during low, moderate, and maximal sustained isometric contractions. Am J Physiol Regul Integr Comp Physiol. 2015; 309: R475-R481. https://goo.gl/AozWqc

4. Sjogaard G, Savard G, Juel C. Muscle blood flow during isometric activity and its relation to muscle fatigue. Eur J Appl Physiol Occup Physiol. 1988; 57: 327-335. Ref.: https://goo.gl/53U3bd

5. Vollestad NK, Wesche J, Sejersted OM. Gradual increase in leg oxygen uptake during repeated submaximal contractions in humans. J Appl Physiol. 1990; 68: 1150-1156. Ref.: https://goo.gl/Uo62ve

6. Wesche J. The time course and magnitude of blood flow changes in the human quadriceps muscles following isometric contraction. J Physiol. 1986; 377: 445-462. https://goo.gl/x6eyPe

7. Hughson RL, Shoemaker JK, Tschakovsky ME, Kowalchuk JM. Dependence of muscle VO2 on blood flow dynamics at onset of forearm exercise. J Appl Physiol. 1996; 81: 1619-1626. Ref.: https://goo.gl/rmjvnb 
8. Wolf M, Ferrari M, Quaresima V. Progress of near-infrared spectroscopy and topography for brain and muscle clinical applications. J Biomed Opt. 2007; 12: 062104. Ref.: https://goo.gl/q4VbzR

9. Hamaoka T, McCully KK, Quaresima V, Yamamoto K, Chance B. Near-infrared spectroscopy/imaging for monitoring muscle oxygenation and oxidative metabolism in healthy and diseased humans. $J$ Biomed Opt. 2007; 12: 062105. Ref.: https://goo.gl/VsJ8Cc

10. Gerovasili V, Dimopoulos S, Tzanis G, Anastasiou-Nana M, Nanas S. Utilizing the vascular occlusion technique with NIRS technology. International Journal of Industrial Ergonomics. 2010; 40: 218-222. Ref.: https://goo.gl/rdPkpu 